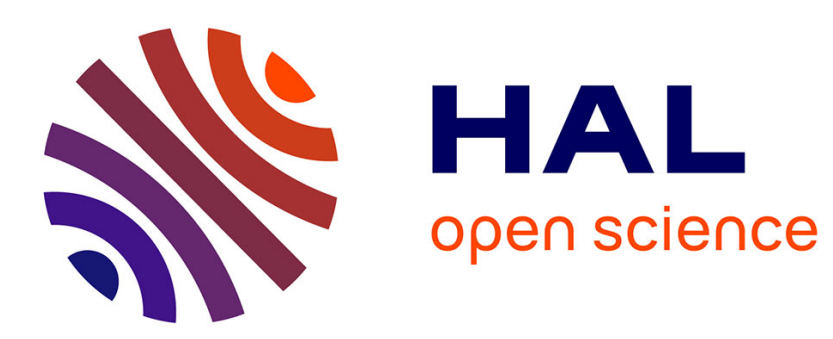

\title{
Composition corporelle et caractéristiques biologiques des muscles chez les bovins en croissance et à l'engrais
}

\author{
Didier Micol, J. Robelin, Y. Geay
}

\section{To cite this version:}

Didier Micol, J. Robelin, Y. Geay. Composition corporelle et caractéristiques biologiques des muscles chez les bovins en croissance et à l'engrais. Productions Animales, 1993, 6 (1), pp.61-69. hal-00896043

\section{HAL Id: hal-00896043 https://hal.science/hal-00896043}

Submitted on 1 Jan 1993

HAL is a multi-disciplinary open access archive for the deposit and dissemination of scientific research documents, whether they are published or not. The documents may come from teaching and research institutions in France or abroad, or from public or private research centers.
L'archive ouverte pluridisciplinaire HAL, est destinée au dépôt et à la diffusion de documents scientifiques de niveau recherche, publiés ou non, émanant des établissements d'enseignement et de recherche français ou étrangers, des laboratoires publics ou privés. 
INRA Prod. Anim., 1993, 6 (1), 61 - 69 .
D. MICOL, J. ROBELIN, Y. GEAY

INRA Laboratoire Croissance et Métabolismes des Herbivores, Theix 63122 Saint-Genès-Champanelle

\section{Composition corporelle et caractéristiques biologiques des muscles chez les bovins en croissance et à l'engrais}

La filière de la viande bovine cherche à maîtriser la qualité de ses produits. Cet article a pour but de résumer les possibilités de modifications de la composition corporelle des animaux (parts des différents tissus à l'abattage) et des caractéristiques biologiques des muscles (couleur, conjonctif, types de fibres musculaires...) qui déterminent en partie la qualité finale des viandes.

La valorisation commerciale des productions de viande bovine est sous la double dépendance de critères quantitatifs (poids) et de critères qualitatifs liés à la composition de la carcasse et aux caractéristiques des muscles. La qualité de la carcasse dépend surtout des proportions relatives de muscles et de dépôts adipeux qu'elle contient, l'objectif étant de maximiser la part de muscle et de limiter au minimum le tissu adipeux. La production des bovins à viande, par différentes approches (génétique, mode de conduite, alimentation) cherche à atteindre cet objectif au moyen du contrôle biologique et zootechnique des différents systèmes de production. Afin de dégager les possibilités de maîtrise, cet article abordera les lois biologiques de variations de la composition corporelle des bovins et de leurs caractéristiques musculaires et il précisera les effets attendus des facteurs zootechniques.

\section{Résumé}

Cet article présente de façon synthétique les connaissances acquises par différentes équipes de recherches sur les lois biologiques de variations de la composition corporelle des bovins et les effets des facteurs zootechniques qui permettent de la modifier (génotype, sexe, stade d'abattage, niveau d'alimentation, nature de la ration et facteurs de croissance). Les caractéristiques du tissu musculaire, déterminantes pour la qualité de la viande, et leurs variations sous l'influence des mêmes facteurs sont également développées.

\section{1 / Croissance et développement des tissus}

La croissance pondérale d'un animal résulte du développement en poids de chacun des éléments constitutifs de son corps. La composition corporelle de l'animal reflète la part relative de chacun de ces éléments. Ainsi, la composition anatomique correspond à la part des différents tissus de la masse corporelle (muscles, dépôts adipeux, os) et des éléments du cinquième quartier (organes, peau, tractus digestif). La composition chimique exprime l'importance relative des différents éléments chimiques (eau, protéines, lipides, minéraux) contenus dans l'ensemble des tissus. La composition corporelle de l'animal se modifie selon le développement différentiel de chacun des éléments. Une des évolutions les mieux connues est celle liée à l'âge et au poids des animaux. Les différents types d'animaux (génotype, sexe...) conduisent à des compositions différentes. Des facteurs d'élevage (systèmes de production, courbes de croissance, vitesses de croissance retenues, niveau d'alimentation, facteurs de croissance...) permettent de modifier le développement des tissus.

\section{1 / Evolution de la composition corporelle avec le poids et l'âge}

Ces lois seront explicitées à partir d'exemples correspondant aux conditions françaises de production. La figure 1 illustre cette évolution chez un jeune bovin mâle 
Figure 1. Evolution de la composition corporelle d'un jeune bovin laitier durant la période de croissance et $d^{\prime}$ engraissement (200 - $550 \mathrm{~kg}$ de poids vif).

Evolution du poids des principaux éléments

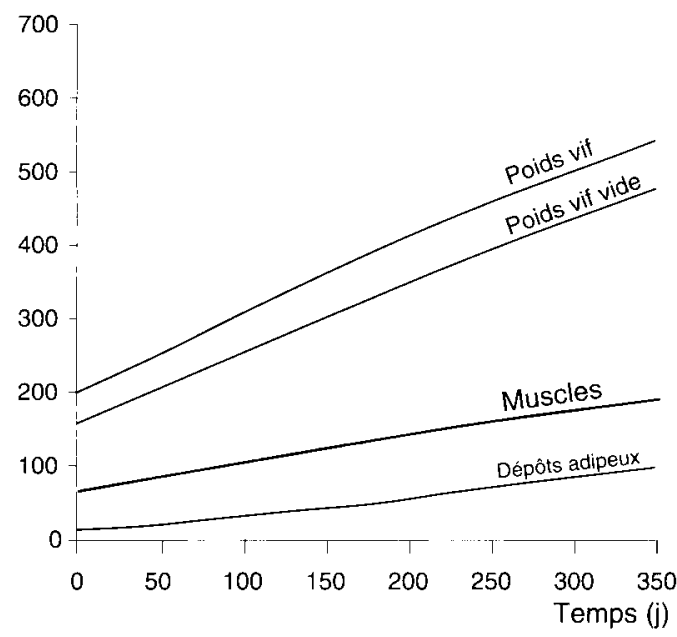

Croît des principaux éléments $(g / j)$

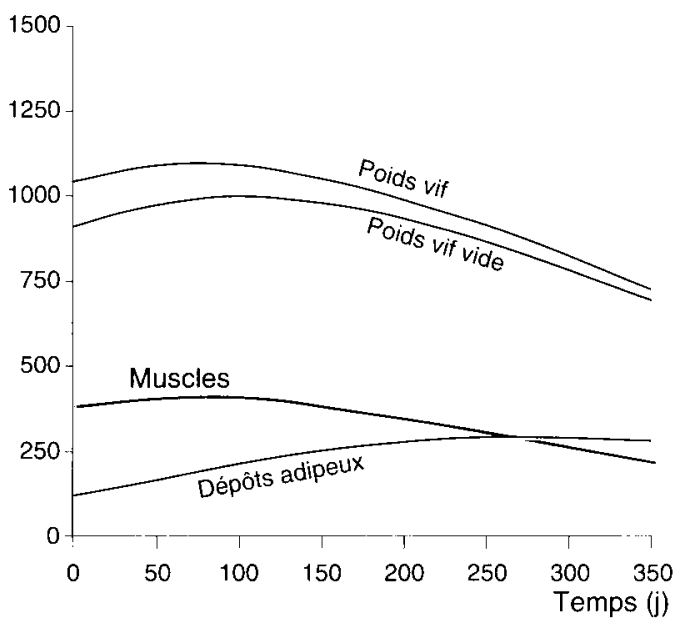

entier Pie Noir à l'engrais entre 200 et $550 \mathrm{~kg}$ de poids vif (durée de $360 \mathrm{j}$ ). L'ensemble de la musculature, essentiellement contenue dans la carcasse, représente la part la plus importante de la masse corporelle (poids vif vide). Chez ce type d'animal, par exemple, le tissu musculaire augmente de 68 à $193 \mathrm{~kg}$, sa part dans la masse corporelle décroît de 43 à $40 \%$ au cours de la période d'engraissement. En effet le dépôt de muscles, limité chez ce type d'animal, décroît avec l'âge. La proportion de muscles dans le gain de la masse corporelle atteint $43 \%$ au début et se réduit à $32 \%$ à l'abattage.

Le tissu osseux représente $17 \%$ de la masse corporelle chez le jeune animal et environ $12 \%$ à la fin de l'engraissement. Les dépôts adipeux ont une importance limitée chez le jeune animal $(12 \mathrm{~kg})$ et se développent rapidement ensuite pour atteindre $100 \mathrm{~kg}$ à l'abattage. Ainsi ces dépôts représentent seulement $7 \%$ de la masse corporelle au départ et atteignent
Composition en $\%$ du poids vif vide

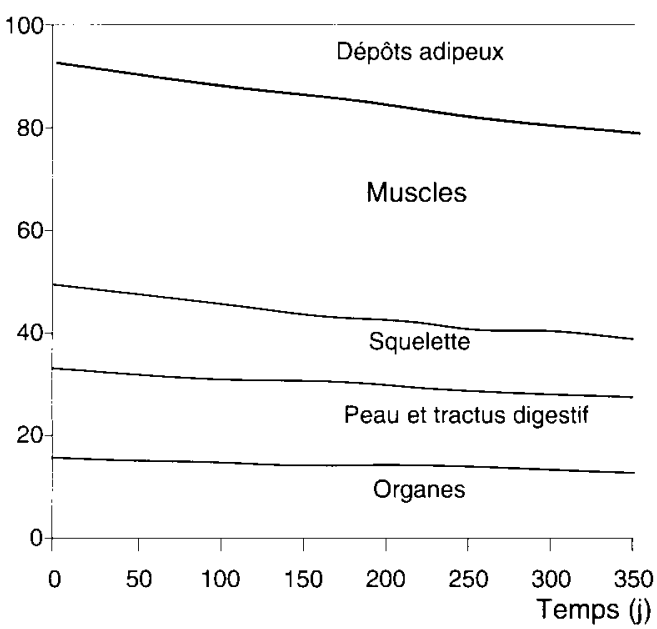

Croît des principaux éléments en $\%$ du croît du poids vif vide

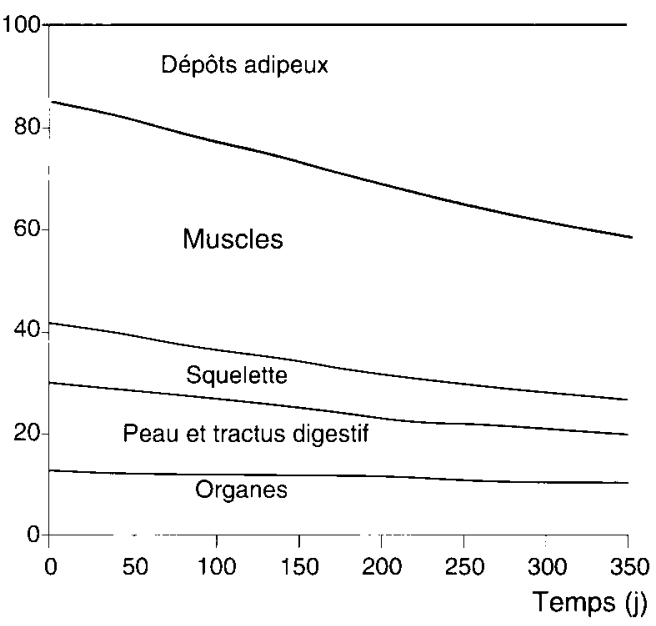

$21 \%$ à $550 \mathrm{~kg}$ de poids vif. Le croît des dépôts adipeux devient de plus en plus important, il atteint dans cet exemple plus de $40 \%$ du gain de la masse corporelle au poids final. A ce stade, chez cet animal, le croît du tissu adipeux est supérieur à celui des muscles $(40$ contre $32 \%$ du gain de masse corporelle.

\section{2 / Influence du génotype et du sexe}

L'évolution générale du développement des différents tissus est analogue chez les différents types de bovins à viande. Cependant on observe des écarts importants du développement des tissus selon leur potentiel de synthèse et selon la rapidité de cette évolution avec l'âge (précocité). Ces variations prennent une importance notable entre animaux de génotypes et de sexe différents. Les comparaisons entre génotypes des performances de croissance et de composition de carcasse à l'abattage ont été rassemblées par différents auteurs 
Tableau 1. Différences entre génotypes Pie Noir (PN) et Limousin (LI) à l'abattage selon différents critères de comparaison (Geay et Robelin 1979).

\begin{tabular}{|l|cc|cc|cc|cc|}
\hline $\begin{array}{l}\text { Critères de comparaison } \\
\text { à l'abattage }\end{array}$ & \multicolumn{2}{|c|}{$\begin{array}{c}\text { Poids } \\
\text { vif } \\
\text { (kg) }\end{array}$} & \multicolumn{2}{c|}{$\begin{array}{c}\text { Poids de } \\
\text { carcasse } \\
\text { (kg) }\end{array}$} & \multicolumn{2}{c|}{$\begin{array}{c}\text { Poids de } \\
\text { muscles } \\
\text { (kg) }\end{array}$} & \multicolumn{2}{c|}{$\begin{array}{c}\text { dépôts } \\
\text { adipeux } \\
\text { \% }\end{array}$} \\
\hline & PN & LI & PN & LI & PN & LI & PN & LI \\
Même âge (16 mois) & 500 & 540 & 274 & 335 & 185 & 258 & 17,9 & 11,3 \\
Même poids vif & $\mathbf{5 5 0}$ & $\mathbf{5 5 0}$ & 303 & 341 & 203 & 262 & 19,3 & 11,4 \\
Même poids de carcasse & 545 & 486 & $\mathbf{3 0 0}$ & $\mathbf{3 0 0}$ & 201 & 231 & 19,2 & 10,6 \\
Même état d'engraissement & 318 & 597 & 169 & 372 & 119 & 285 & $\mathbf{1 2 , 0}$ & $\mathbf{1 2 , 0}$ \\
Même stade (1) & 540 & 600 & 297 & 374 & 199 & 287 & 19,0 & 12,1 \\
Même efficacité alimentaire & 425 & 600 & 230 & 374 & 158 & 287 & 15,5 & 12,1 \\
\hline
\end{tabular}

(1) même stade physiologique ; $60 \%$ du poids adulte

(2) $180 \mathrm{~g}$ de gain de poids par UF, de $300 \mathrm{~kg}$ PV à l'abattage

Tableau 2. Gains journaliers estimés de tissus selon le sexe chez des bovins de race tardive conduits aux mêmes poids et gain de poids vif journalier (d'après Robelin 1990).

\begin{tabular}{|l|c|c|c|}
\hline Sexe & Mâle entier & Mâle castré & Femelle \\
\hline Gain de poids vif $(\mathrm{g} / \mathrm{j})$ & 1000 & 1000 & 1000 \\
Gain de dépôts & & & \\
adipeux totaux $(\mathrm{g} / \mathrm{j})$ & 138 & 193 & 303 \\
Gain de muscles $(\mathrm{g} / \mathrm{j})$ & 469 & 448 & 384 \\
\hline
\end{tabular}
Les génotypes à fort
potentiel de croissance musculaire ont des durées d'engraissement plus longues et présentent des états d'engraissement moindres que les génotypes laitiers plus précoces.

(Geay et Micol 1988 ; Bass et al 1990), et mettent en évidence des différences importantes de développement des tissus entre génotypes. Le tableau 1 illustre ces différences entre des jeunes bovins mâles entiers très précoces (Pie Noir) ou tardifs (Limousin). Quel que soit le critère de comparaison, le génotype tardif à fort potentiel de croissance musculaire présente une supériorité de production de carcasse et de muscles tout en gardant un état d'engraissement limité. Ainsi, dans la pratique, ces génotypes ont des durées d'engraissement plus longues et présentent des états d'engraissement moindres que leur croisement avec d'autres génotypes ou que les génotypes laitiers plus précoces.

Le sexe de l'animal se traduit dans la pratique par des compositions corporelles différentes. Les femelles ont un potentiel de croissance pondérale plus faible que celui des mâles entiers, mais un développement plus rapide des tissus adipeux. Ainsi, à même poids vif, les femelles présentent un poids plus élevé de dépôts adipeux. Les mâles castrés ont une croissance pondérale et une vitesse de développement intermédiaires entre celles des mâles entiers et celles des génisses. Ces différences ont été étudiées sur différents types et génotypes de bovins. Elles ont été rassemblées par différents auteurs (Robelin et Geay 1984 ; Bass et al 1990). Le tableau 2 illustre les gains journaliers prévisibles de tissus selon le sexe, à partir d'un modèle de simulation des phénomènes de croissance, chez des animaux de race à viande.

\section{3 / Influence du stade et du poids à l'abattage}

Comme indiqué précédemment, le tissu adipeux a un développement de plus en plus rapide avec l'augmentation du poids de l'animal durant sa finition. La croissance musculaire diminue dans le même temps. Ainsi, le choix du poids à l'abattage permet de moduler la part relative des différents tissus. Pour un même niveau de croissance durant la finition, une réduction du poids final retenu se traduit par une diminution de l'état d'engraissement; une augmentation du poids a l'effet contraire. C'est ce phénomène qui, dans la pratique, préside à la décision d'abattage des bovins. La figure 2 illustre l'effet d'une modification du poids à l'abattage autour de $550 \mathrm{~kg}$ sur le poids des tissus déposés et sur leur part dans la masse corporelle (poids vif vide) chez un jeune bovin précoce à l'engrais (Pie Noir). Pour une augmentation de $50 \mathrm{~kg}$ du poids à l'abattage, le tissu adipeux augmente de $21 \mathrm{~kg}$ soit un accroissement relatif de $23 \%$; alors que les muscles s'accroissent de seulement $8 \%$ (15 kg). La composition de la masse corporelle à l'abattage reflète ces évolutions (20 vs $22 \%$ de dépôts adipeux entre 525 et $575 \mathrm{~kg}$ de poids vif à l'abattage).

L'évolution de la composition corporelle est cependant moins rapide chez les animaux tardifs et chez les bovins abattus à un stade physiologique moins avancé. A titre d'exemple, un même écart de $50 \mathrm{~kg}$ du poids à l'abattage d'un mâle Charolais de $700 \mathrm{~kg}$ (Micol et Robelin 
Figure 2. Variations de la composition corporelle d'un jeune bovin laitier selon le poids à l'abattage.

Poids des principaux éléments $(\mathrm{kg})$

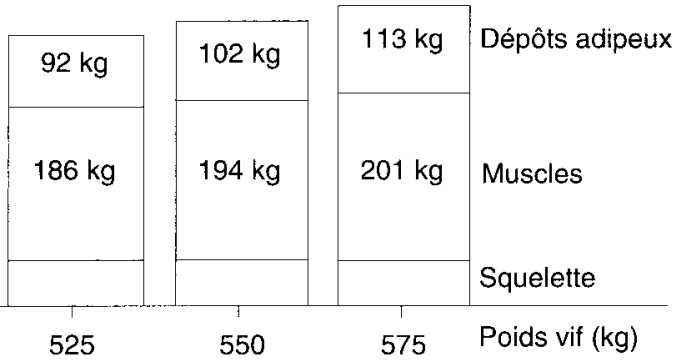

Composition en $\%$ du poids vif vide

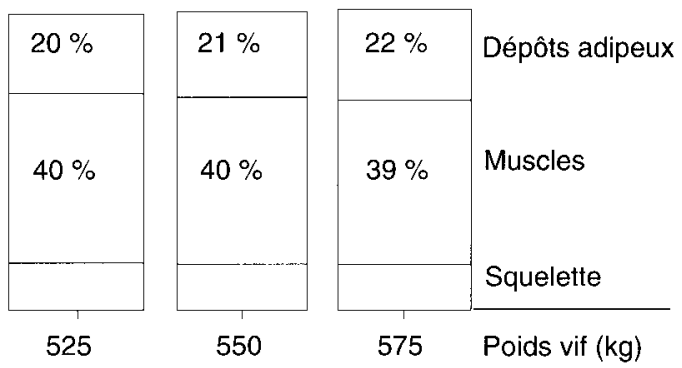

1990) se traduit par une augmentation du poids de muscles de $24 \mathrm{~kg}(+8 \%)$ et de seulement $11 \mathrm{~kg}$ du tissu adipeux $(+13 \%)$. Ces données mettent en évidence les possibilités de maîtriser l'état d'engraissement des bovins à viande par le choix approprié de la durée de finition et de l'objectif de poids à l'abattage.

\section{4 / Influence de la vitesse de croissance et du niveau d'alimentation}

Les variations du niveau des apports alimentaires, énergétiques principalement, permettent de modifier la vitesse de croissance pondérale permise, la composition du croît des bovins et, par voie de conséquence, la composition corporelle à l'abattage. Ces modifications s'expliquent par le fait que les parts respectives de lipides et de protéines déposées varient selon le niveau d'alimentation retenu. Globalement, lorsque l'apport énergétique s'élève, la quantité de lipides déposée augmente d'autant plus vite que le gain de poids s'accroît (Robelin et Daenicke 1980). En conséquence, les quantités déposées de tissus adipeux et musculaire vont refléter ces modifications de la composition chimique du croît de l'animal.

La figure 3 illustre ces variations en quantités et en proportions de tissus lorsque le gain de poids vif s'accroît $(0,6$ à $1,2 \mathrm{~kg} / \mathrm{j})$ du fait du niveau d'alimentation chez un mâle entier précoce (Pie Noir). Les quantités journalières de
Figure 3. Influence du gain de poids journalier sur la composition du croît d'un jeune bovin laitier de $500 \mathrm{~kg}$ de poids vif.

Croît journalier des tissus $(g / j)$

Part dans le gain de poids vif vide en \%

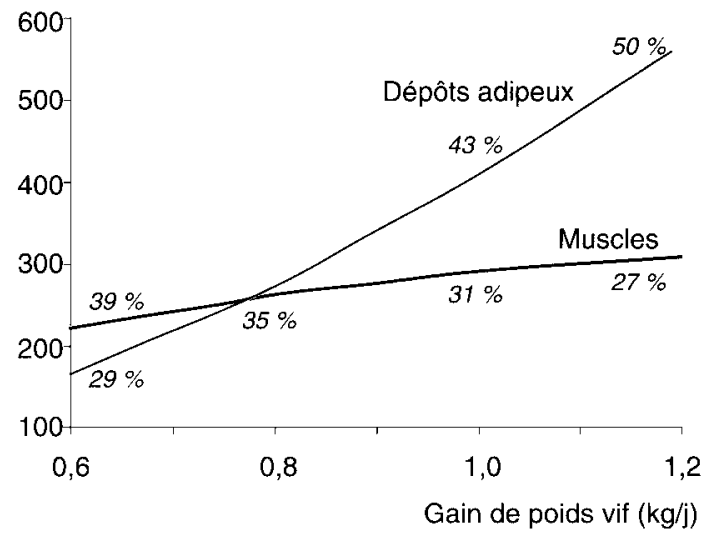

Figure 4. Poids des tissus déposés au cours de l'engraissement d'un jeune bovin laitier selon la vitesse de croissance moyenne retenue $(0,8$ et $1,0 \mathrm{~kg} / \mathrm{j})$.

Poids des tissus $(\mathrm{kg})$

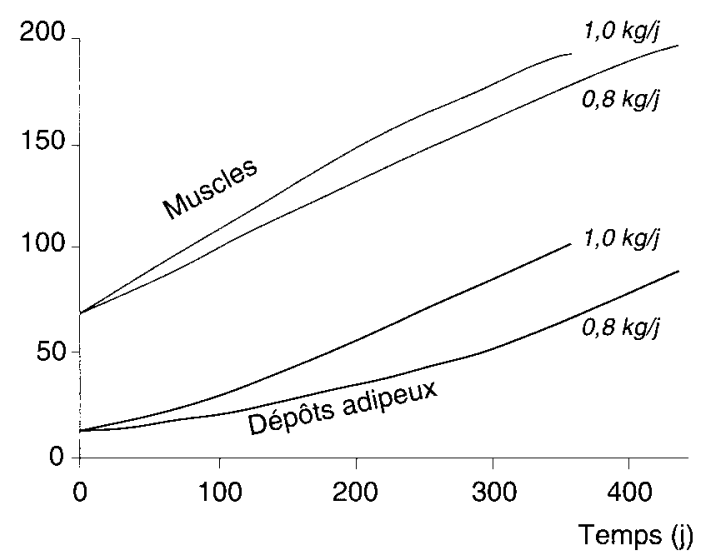

tissu musculaire formé augmentent avec le gain mais moins vite que les dépôts adipeux. Ainsi le dépôt de muscles qui représente $39 \%$ $\mathrm{du}$ gain de masse corporelle pour $0,6 \mathrm{~kg} / \mathrm{j}$ n'en représente plus que $27 \%$ pour $1,2 \mathrm{~kg} / \mathrm{j}$. Pour la même augmentation de la vitesse de croissance, les dépôts adipeux ont un développement préférentiel, ils occupent $29 \%$ (164 g) du gain de masse corporelle pour $0,6 \mathrm{~kg} / \mathrm{j}$ et $50 \%$ $(570 \mathrm{~g})$ pour une croissance de $1,2 \mathrm{~kg} / \mathrm{j}$.

Sur l'ensemble de la période d'engraissement, le choix du gain de poids journalier à réaliser et donc du niveau alimentaire permet de moduler la formation des différents tissus et d'aboutir à l'abattage à des compositions corporelles distinctes. La figure 4 illustre cette application avec deux niveaux moyens de croissance $(0,8$ et $1,0 \mathrm{~kg} / \mathrm{j})$ chez un jeune mâle Pie Noir abattu à $550 \mathrm{~kg}$ dans les deux cas. La réduction de la croissance pondérale moyenne se traduit par un allongement de la phase de finition $(75 \mathrm{j})$ pour atteindre les mêmes poids vifs à l'abattage et de carcasse. Le gain de poids modéré entraîne une limitation progressive des tissus adipeux à l'abattage ; $90 \mathrm{~kg}$ 
contre $102 \mathrm{~kg}$ chez l'animal plus alimenté, soit une réduction relative de $12 \%$ de l'état d'engraissement. A l'inverse la limitation des apports alimentaires favorise le croît des tissus musculaires, $198 \mathrm{~kg}$ contre $192 \mathrm{~kg}$.

Le niveau des apports alimentaires interagit cependant avec la précocité et le stade de développement physiologique de l'animal (Micol et Robelin 1990). La limitation du niveau d'alimentation est d'autant plus intéressante que l'animal est précoce et développe beaucoup ses tissus adipeux. Au contraire, la réduction du niveau d'alimentation chez un animal tardif se traduit par une réduction importante du gain de poids vif et de sa synthèse protéique sans modifier notablement le tissu adipeux, déjà limité chez ce type d'animal. Ces interactions entre le génotype et le niveau d'alimentation ont fait l'objet de nombreuses études dans le but de définir les modes de conduite les mieux adaptés au potentiel de l'animal tout en maîtrisant la qualité de la carcasse et le coût de production (Geay et Robelin 1979).

\section{5 / Influence de la nature de la ration}

De nombreuses expérimentations ont été réalisées pour mettre en évidence les effets de la quantité et de la nature des apports de la ration sur le développement des tissus et la composition corporelle à l'abattage. Les variations des apports énergétiques (Cf 1.4) permettent de modifier les parts respectives de lipides et de protéines dans le gain de poids (Robelin et Daenicke 1980). Les effets des quantités d'énergie ingérées sur la composition corporelle ont été décrits pour différents types d'animaux et différents génotypes (Geay et Robelin 1979 ; Robelin et Geay 1984 ; J. Bass 1990). Ces effets, maintenant mieux connus, ont permis d'établir des recommandations d'apports énergétiques et azotés selon le type d'animal et la composition du gain recherchée (Geay et al 1987).

Le niveau d'apports protéiques permet de moduler le développement des différents tissus chez le monogastrique (cf revue de Bass 1990). Son effet est beaucoup moins sensible chez le ruminant, du moins autour et au delà de la satisfaction des besoins nutritionnels azotés. Ces résultats engagent à mieux étudier les effets des équilibres énergie/azote (PDI/UF) de la ration et de la nature des nutriments fournis (acides aminés) sur la composition du gain des bovins et sur la maîtrise de la composition des carcasses (Micol et Robelin 1990).

\section{6 / Influence des facteurs de croissance}

Les facteurs de croissance (antibiotiques, hépatoprotecteurs, agents anabolisants) sont utilisés avec l'objectif prioritaire d'accroître l'efficacité de la production de viande chez les bovins. Les antibiotiques et les hépatoprotecteurs à activité digestive et/ou métabolique ne semblent pas modifier notablement le développement relatif des tissus de l'animal (Geay 1990, Owens et al 1991). Par contre les sub- stances à activités hormonales ont des effets directs sur les quantités et la répartition des tissus formés.

Les effets des stéroïdes ont largement été étudiés selon différentes formes et doses d'administration chez divers types d'animaux et les possibilités d'accroissement des poids de carcasse et de muscles produits tout en limitant les dépôts de tissu adipeux ont été montrées (cf revues Geay, 1984 ; Bass 1990 ; Fiems et al 1991 ; Hancock et al 1991). Leur utilisation se heurte dans la pratique à leur interdiction légale d'emploi, dans les pays de la CEE notamment.

Les hormones de l'axe somatotrope suscitent un grand intérêt actuellement en raison notamment des possibilités de fabrication par recombinaison génétique. La figure 5 résume les essais d'administration de $\mathrm{GH}$ sur des bovins à viande (Geay 1990). L'effet du traitement à la GH se traduit par une amélioration moyenne des vitesses de croissance de $12 \%$, une augmentation proportionnelle des poids de carcasse obtenus et, pour certains essais, un accroissement de la masse musculaire produite.

Les applications basées sur l'utilisation d'autres hormones de l'axe somatotrope (GHRF, SRIF, IGF) nécessitent de mieux connaitre leurs mécanismes d'action (Beermann et Devol 1991). La variabilité des procédés et des réponses ne permet pas de dégager leurs effets quantitatifs sur le développement des différents tissus chez les bovins producteurs de viande (Bass 1990 ; Geay 1990).

Les $ß$-agonistes des catécholamines ont montré ces dernières années leur possibilité d'accrô̂tre la synthèse protéique et de diminuer la lipogénèse et d'aboutir ainsi à des différences notables de composition corporelle des animaux à viande. La synthèse des essais réalisés chez les bovins fait ressortir une amélioration, dose-dépendante, du gain de poids et de l'efficacité alimentaire, une augmentation de 4

Figure 5. Effets de l'hormone de croissance exogène sur la croissance, lingestion et l'état d'engraissement des bovins (Geay 1990).

en $\%$ du témoin

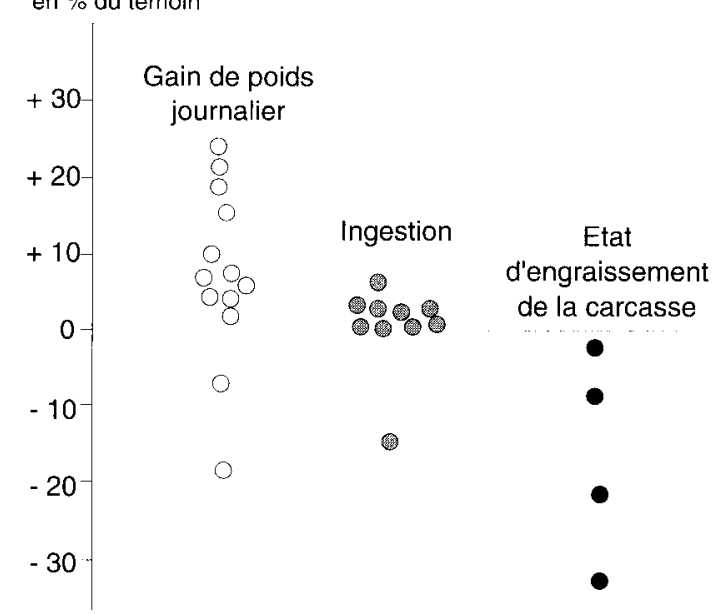

L'augmentation du niveau d'alimentation accroît la part des lipides totaux dans le gain de poids 
à 5 points du rendement en carcasse, un accroissement de la masse musculaire $(+9$ à $17 \%$ ) et une réduction de la masse adipeuse pouvant atteindre $30 \%$ (Geay 1990 ; Fiems et al 1991). Il existe cependant diverses molécules dont les effets sont à préciser aussi bien sur le développement des tissus que sur les caractéristiques biologiques des muscles et des viandes (cf revue de Moloney et al 1991).

\section{2 / Caractéristiques biologiques des muscles}

La qualité de la viande est sous la dépendance des caractéristiques des muscles et des facteurs technologiques appliqués après l'abattage. Les variations entre muscles d'un même animal sont importantes (composition, couleur, importance et nature du tissu conjonctif, types des fibres musculaires constitutives) et président à leur utilisation ultérieure en tant que morceaux par la filière commerciale et le consommateur. Cependant les facteurs zootechniques liés à la production de l'animal (âge, sexe, génotype, mode de conduite) agissent également sur ces caractéristiques et permettent de les maîtriser dans une certaine mesure.

\section{1 / Influence de l'âge}

La composition chimique du muscle (lipides et protéines) suit l'évolution de l'ensemble de la masse corporelle, c'est-à-dire un accroissement de la teneur en lipides avec l'âge (Robelin 1986) mais cet accroissement reste faible pour le tissu musculaire comparativement à l'augmentation des dépôts adipeux (figure 6). Dans le cas des animaux étudiés, jeunes bovins mâles entiers, les valeurs atteintes ne dépassent pas 4 à $5 \%$ du muscle frais pour un poids

Figure 6. Evolution de la teneur en lipides intramusculaires avec l'état d'engraissement chez de jeunes bovins mâles de différents génotypes: Charolais (Ch), Limousin (Li), Salers (SI), et Pie Noir (PN) (d'après Robelin 1978).

Lipides intramusculaires en \% du poids des muscles frais de la carcasse

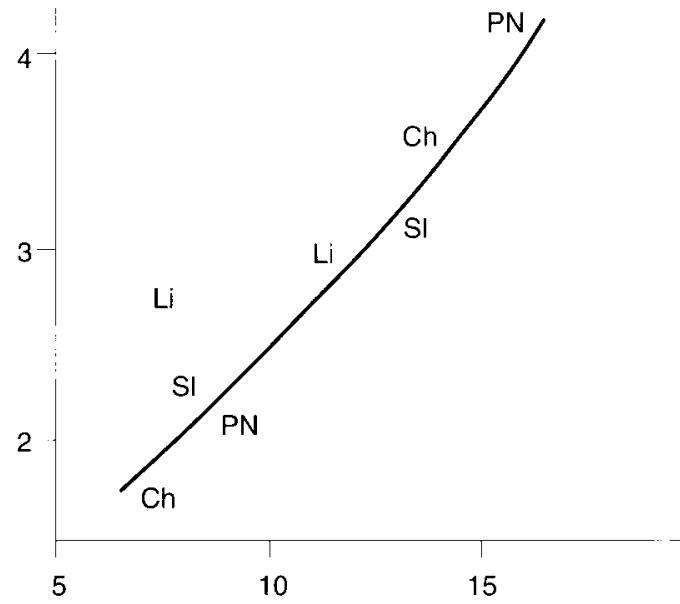

Dépôts adipeux totaux en \% du poids vif vide total des tissus adipeux d'environ $15 \%$ de la masse corporelle (Robelin et Daenicke 1980). Parallèlement, on observe une légère diminution des teneurs en protéines et en eau des muscles. Cependant, ces variations deviennent très faibles (légère augmentation des protéines), au moins durant la période post natale, si elles sont rapportées à la masse délipidée (Robelin 1986).

L'évolution avec l'âge des caractéristiques physico-chimiques des muscles (composition, tissu conjonctif, tendreté, coloration) a été étudiée à partir de jeunes bovins mâles entiers entre 9 et 24 mois d'âge (Kopp 1982). L'intensité de la couleur augmente avec l'âge en raison de l'augmentation du taux de myoglobine. Cette teneur s'accroît rapidement jusqu'à l'âge de 16-18 mois, l'augmentation devient plus modérée ensuite. La stabilité de la couleur tend à diminuer avec l'âge surtout dans les muscles les plus sensibles à la stabilité de la couleur. La teneur en collagène des muscles augmente de 18 à $20 \%$ entre 9 et 13 mois d'âge, au delà de 13 mois les muscles présentent de faibles variations de cette teneur. Cependant la solubilité du collagène, reflet de son degré de polymérisation, diminue progressivement et explique la diminution de la tendreté des muscles avec l'âge de l'animal.

Le nombre de fibres musculaires est fixé dès la fin de la vie foetale chez le bovin, cependant la quantité de DNA (nombre de noyaux) s'accroît d'un facteur de 3 à 5 durant la vie postnatale. Ainsi, la croissance musculaire postnatale est due à un accroissement du diamètre des fibres, qui passe de $30 \mu$ à la naissance à 70-100 $\mu$ chez l'adulte. Les fibres musculaires se mettent en place durant la vie fœtale selon 4 types : type I (lent oxydatif), type IIa (rapide oxydoglycolytique), type IIb (rapide glycolytique) et type IIc (type foetal ayant simultanément des myosines lente et rapide) (Robelin 1990 b). Durant le début de la vie postnatale, les fibres de type IIc se transforment en partie en fibres de type I dont le pourcentage augmente. Au delà de cette période, le pourcentage de ce type de fibres reste stable, tandis que le pourcentage des fibres de type IIa diminue au profit des fibres IIb (figure 7). Cette évolution traduit ainsi une diminution avec l'âge du métabolisme oxydatif au niveau musculaire.

\section{2 / Influence du sexe}

La teneur en lipides intramusculaires est liée à la teneur en lipides du corps entier, on retrouve ainsi à ce niveau les effets des différences de précocité liées au sexe de l'animal

La teneur en pigments croît plus vite chez la femelle que chez le mâle entier, l'augmentation est d'autant plus rapide que les muscles sont plus colorés, par contre, ces différences ne semblent pas se manifester aussi nettement entre bovins mâles entiers et castrés (Renerre 1986).

L'influence du sexe sur le tissu conjonctif est connue et correspond à des teneurs et des solubilités différentes du collagène. Le taux de collagène est plus élevé chez les mâles entiers 
Figure 7. Evolution avec l'âge des différents types de fibres dans les muscles squelettiques des bovins (d'après Ashmore et al 1972, Holmes et al 1972; Solomon et al 1986).

Proportion des différents types de fibres (\% du total)

IIB

50

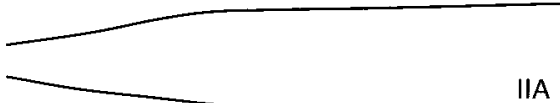

25 $\uparrow 3$ mois

Naissance

que chez les génisses, les mâles castrés occupent une position intermédiaire. La solubilité du collagène est similaire chez les castrés et les femelles, elle est plus faible chez les animaux entiers (Monin 1991). Ces différences sont en interaction avec l'âge des animaux : relativement faibles chez les jeunes animaux, elles s'accroissent avec l'âge (Kopp 1982).

Des différences entre les types de fibres musculaires se retrouvent également selon le sexe des animaux. Les muscles des mâles entiers présentent un pourcentage plus important de fibres lentes oxydatives et moins important de fibres rapides glycolytiques que ceux des bovins castrés (Seideman et Crouse 1986). Généralement, pour un même type, les diamètres des fibres chez les mâles sont supérieurs à ceux des animaux castrés et des femelles. Les études en cours visent à préciser les effets des hormones sexuelles sur ces phénomènes.

\section{3 / Influence du génotype}

La teneur en lipides des muscles varie nettement entre génotypes en liaison avec leur précocité, ce qui peut contribuer aux variations génotypiques de la tendreté. Cependant, à même état d'engraissement de la masse corporelle, il ne semble pas y avoir de différence dans la teneur en lipides intramusculaires selon le génotype (Robelin 1978).

Des différences génotypiques dans la teneur en pigments ont été rapportées ; l'intensité de la couleur tend à varier inversement au développement musculaire.

La teneur en collagène du muscle diminue également avec le développement musculaire, en interaction avec le stade physiologique, selon les génotypes, ce qui peut également contribuer aux différences de tendreté (Boccard et al 1979; Boccard et Bordes 1986). Renand (1988) en analysant les relations génétiques entre la croissance musculaire et les qualités de la viande conclut cependant au manque de liaisons claires entre ces caractéristiques.
Les différences éventuelles des types de fibres musculaires selon le génotype sont encore mal connues. Une proportion plus importante de fibres rapides a été mise en évidence avec le gène culard, variation associée à des modifications de la quantité et du type de collagène et à des altérations de la couleur et des lipides intramusculaires. Ces différences peuvent laisser supposer des variations des types de fibres musculaires entre les génotypes et leurs conséquences sur la qualité des muscles (May et al 1975).

\section{4 / Influence du niveau d'alimentation et de la nature de la ration}

L'augmentation du niveau d'alimentation accroît la part des lipides totaux dans le gain de poids (cf 1.4). Robelin et Daenicke 1980 observent une augmentation moyenne de $18 \%$ des lipides totaux déposés pour une augmentation de $10 \%$ de la vitesse de croissance. Ces valeurs demandent à être précisées au niveau des lipides et des protéines des muscles dans différentes conditions.

Il existe assez peu de données sur les effets intrinsèques de la vitesse de croissance des animaux sur d'autres caractéristiques biologiques des muscles. Pour certains auteurs, l'augmentation du niveau d'alimentation conduirait à une baisse de la teneur en collagène (Miller et al 1987) ; pour d'autres il n'aurait pas d'effet sur le tissu conjonctif et la couleur (Boccard et Bordes 1986). Par contre, le mode d'élevage (forme de courbe de croissance) peut modifier le tissu conjonctif. Ainsi Kopp (1982) met en évidence une solubilité du collagène plus importante chez des mâles entiers de deux ans ayant pâturé avant leur finition à l'auge que chez des mâles plus jeunes conduits à l'auge depuis le sevrage.

La nature de l'alimentation semble peu influencer les caractéristiques des muscles des animaux pour peu que les comparaisons soient effectuées à des états de développement et d'engraissement voisins à l'abattage. Cependant des études sont actuellement engagées à l'INRA sur les effets éventuels de l'alimentation azotée et protéique (acides aminés) sur les caractéristiques musculaires des bovins.

\section{5 / Influence des facteurs de croissance}

L'emploi des agents anabolisants (stéroïdes) se traduit par une diminution de la teneur en lipides des muscles (Geay et al 1986 ; Ouali 1991). La solubilité du collagène semble diminuer et la teneur en fer héminique augmenter avec l'emploi des anabolisants (Ouali 1991). Les caractéristiques des fibres musculaires sont également affectées. Clancy (1986) rapporte une diminution des fibres rapides glycolytiques. L'ensemble de ces modifications peuvent expliquer en partie la dégradation des qualités des viandes (tendreté, jutosité) observée chez les animaux implantés (Geay et al 1986).
La nature de l'alimentation semble peu influencer les caractéristiques des muscles des animaux. 
Les $B$-agonistes semblent modifier notablement les caractéristiques du tissu musculaire (Ouali 1991 ; Wood et al 1990). La teneur en lipides est considérablement diminuée. Les quantités de collagène et de fer héminique sont également plus faibles. L'histologie du muscle semble être orientée vers une hypertrophie des fibres musculaires et un accroissement des fibres glycolytiques blanches. Cependant ces modifications, qui restent à préciser, semblent avoir des conséquences néfastes sur la qualité des viandes (Wood 1990 ; Moloney et al 1991).

Les effets des hormones de l'axe somatotrope sur les caractéristiques biologiques des muscles et leurs aptitudes à la transformation en viande, chez les bovins, demandent encore à être précisés. Les résultats rapportés par Allen et Enright (1989) montrent cependant une légère tendance à une dégradation de la tendreté du fait d'une diminution de la solubilité du collagène et d'une augmentation de sa teneur.

\section{Conclusion}

Les lois d'évolution de la composition corporelle des bovins et du développement des différents tissus, ainsi que les effets des principaux facteurs de variation, sont de mieux en mieux connus dans différentes situations. Ces connaissances permettent maintenant de simuler et quantifier les effets attendus des différents facteurs zootechniques dans leurs conditions d'application. Ainsi les objectifs de poids finaux et la vitesse de croissance pondérale retenue pour y parvenir sont des outils efficaces pour limiter le développement trop important du tissu adipeux des bovins en interaction avec le type et la précocité de l'animal utilisé. Ces relations demandent cependant à être précisées dans le cadre des formes de courbes de croissance discontinue où il existe des interactions importantes entre les différentes phases de la vie de l'animal.

Certains facteurs de croissance peuvent modifier notablement le développement des différents tissus ; les effets des $\beta$-agonistes et des hormones de l'axe somatotrope sont dans ce domaine à préciser dans différentes situations.

Les caractéristiques biologiques des muscles (teneur en lipides, couleur, tissu conjonctif, types de fibres musculaires) sont mieux connues chez les bovins producteurs de viande. Les facteurs de nature zootechnique (âge, sexe, génotype) peuvent également modifier en partie ces caractéristiques; les effets de certains d'entre eux doivent être précisés pour être appliqués dans la pratique (vitesse de croissance, nature et quantités des apports nutritifs, facteurs de croissance et hormonaux).

Les connaissances sur les effets zootechniques s'organisent de mieux en mieux et permettent d'agir au niveau de la conduite de l'animal et de l'élaboration de ses tissus tout en maîtrisant les répercussions sur les caractéristiques biologiques de ses muscles et en prévoyant les conséquences finales sur les qualités des viandes fournies à la consommation.

\section{Références bibliographiques}

Allen P., Enright W.J. 1989. Effects of administration of somatotropin on meat quality in ruminants : a review. In use of somatotropin in livestock production. Eds K. Sejrsen, M Vestergaard and A. Neimann-Sorensen, Elsevier Applied Science, London, pp 201-209.

Bass J.J., Butler-Hogg B.W., Kirton A. H., 1990 Practical methods of controlling fatness in farm animals. In Reducing fat in meat animals. Eds Wood and Fischer. Elsevier Applied Science, London, pp 145-200.

Beermann D.H., Devol D.L., 1991. Effects of somatotropin, somatotropin realising factor and somatostatin on growth. In Growth regulation in farm animals. Eds A. M. Pearson et T.R. Dutson. Elsevier applied science, Londres. 373-426.

Boccard R., Naude R.T., Cronje D.E., Smit M.C., Venter H.J., Rossouw E.J., 1979. The influence of age, sex and breed of cattle on their muscle characteristics. Meat Sci., 4, 261-281.

Boccard R., Bordes P., 1986. Caractéristiques qualitatives et technologiques des viandes bovines: influence des facteurs de production. In Production de viande bovine. Ed D. Micol, INRA Publ, Paris, pp 61-84.

Clancy M.J., Lester Janet M., Roche J.F., 1986. The effects of anabolic agents and breeds on the fibers of the longissimus muscle of male cattle. J. Anim. Sci., 63, 83-91.

Fiems L.O., Cottyn B.G., Boucqué Ch. V., 1991 Growth promoters and meat yield. In Animal biotechnology and the quality of meat production. Eds L O Fiems, B G Cottyn and D I Demeyer; Elsevier Science Publishers, Amsterdam, pp 31-48.

Geay Y., Robelin J., 1979. Variation of meat production capacity in cattle due to genotype and level of feeding: genotype-nutrition interaction. Liv. Prod. Sci., 6, 263-276

Geay Y., 1986. Emploi des additifs alimentaires et des anabolisants pour la production de viande de gros bovins. In Production de viande bovine. Ed D Micol, INRA Publ, Paris, pp 417-460.

Geay Y., Micol D., Robelin J., Berge Ph., Malterre C., 1987. Recommandations alimentaires pour les bovins en croissance et à l'engrais. Bull. Tech. CRZV Theix, INRA, 70, 173-183.

Geay Y., Micol D., 1988. Utilization of large size cattle breeds in the main fattening systems in continental Europe. Proceedings of 3rd World Congress on sheep and beef cattle breeding. 19-23 June 1988, Paris. Volume 2, INRA Publ., Paris, 113126.

Geay Y., 1990. Diverses possibilités pharmacologiques de modifier la croissance et l'efficacité alimentaire des bovins. In Croissance des bovins et qualité de la viande. Eds R. G. Guilhermet et Y Geay, Rennes, 8-10 Novembre 1989. ENSAR Rennes, France. 87-114.

Hancock D.L., Wagner J.F., Anderson D.B., 1991. Effects of estrogens and androgens on animal growth. In Growth regulation in farm animals. Eds A. M. Pearson et T.R. Dutson. Elsevier applied science, Londres. 255-297. 
Kopp J., 1982. Qualité des viandes de taurillons: évolution avec l'âge des caractéristiques physicochimiques des muscles. Bull. Techn. CRZV Theix INRA, 48, 27-46.

May M.L., Dikeman M.E., Schalles R.R., 1975. Histology of exotic cross bovine muscle. J. Anim. Sci., 41, 298 (Abstract).

Micol D., Robelin J., 1990. Evolution de la composition corporelle et facteurs zootechniques de variation. In Croissance des bovins et qualité de la viande. Eds R. G. Guilhermet et Y Geay, Rennes, 810 Novembre 1989. ENSAR Rennes, France. 15-30.

Miller M.F., Cross H.R., Buyck M.J., Crouse J.D., 1987. Bovine longissimus dorsi glycogen and color response as affected by dietary regimen and post mortem electrical stimulation in young bulls. Meat Sci., 19, 253-262.

Moloney A., Allen P., Joseph R., Tarrant V., 1991. Influence of beta-adrenergic agonists and similar compounds. In Growth regulation in farm animals. Eds A. M. Pearson et T.R. Dutson. Elsevier applied science, Londres. 455-513.

Monin G., 1991. Facteurs biologiques des qualités de la viande bovine. INRA Prod. Anim., 4 (2), 151-160.

Ouali A., 1991. Sensory quality of meat as affected by muscle biochemistry and modern technologies. In Animal biotechnology and the quality of meat production. Eds L O Fiems, B G Cottyn and D I Demeyer. Elsevier Science Publishers, Amsterdam, pp 85-105.

Owens F.N., Zorilla-Rios J., Dubeski P., 1991 Effects of ionophores on metabolism, growth, body compossition and meat quality. In Growth regulation in farm animals. Eds A. M. Pearson et T.R. Dutson. Elsevier applied science, Londres. 321342 .

Renand G., 1988. Genetic determinism of carcass and meat quality in cattle. Proceedings of 3rd World Congress on sheep and beef cattle breeding. 19-23
June 1988, Paris. Volume 1, INRA Publ., Paris, 381395.

Robelin J., 1978. Répartition des dépôts adipeux chez les bovins selon l'état d'engraissement, le sexe et la race. Bull. Techn. CRZV Theix INRA, 34, 31-34.

Robelin J., Daenicke R., 1980. Variation of net requirements for cattle growth with liveweight, liveweight gain, breed and sex. Ann. Zootech., 29 HS, 15-30

Robelin J., Geay Y., 1984. Body composition of cattle as affected by physiological status, breed, sex and diet. Proceedings of International Symposium on Herbivores Nutrition, 5-9 April 1983, Pretoria. The Science Press, pp 525-548.

Robelin J., 1986. Composition corporelle des bovins: Evolution au cours du développement et différences entre races. Thèse d'état, Université de Clermont Ferrand II, E-368, pp 392.

Robelin J., 1990. Modèle de calcul du croît journalier de lipides et de protéines chez les bovins. Reprod. Nutr. Dév., 5ème Journées Alimentation Nutrition des Herbivores, Mars 1989. suppl. 2, 245s-246s.

Robelin J., 1990 b. Différenciation, croissance et développement cellulaire du tissu musculaire. INRA Prod. Anim., 3, 253-263.

Seideman S.C., Crouse J.D., 1986. The effects of sex condition, genotype and diet on bovine muscle fiber characteristics. Meat Sci., 17, 55-72.

Wood J.D., 1990. Consequences for meat quality of reducing carcass fatness. In Reducing fat in meat animals. Eds Wood and Fischer. Elsevier Applied Science, London, pp 344-397.

Cet article reprend une présentation sur l'évolution de la composition corporelle des bovins et sur les caractéristiques biologiques des muscles (D.Micol, J.Robelin, Y. Geay) au Séminaire FEZ "Carcasse et évaluation de la qualité de la viande" à Rostock en Septembre 1991.

\section{Summary}

\section{Growth and development of tissues and biological characteristics of muscle in rearing and finishing beef cattle.}

This paper gives an overview of what the various teams have learnt concerning the biological laws for variation in body composition of cattle and the effects of zootechnical factors influencing this (breed, sex, slaughter weight, feeding level, type of feed and growth factors). The biological characteristics of muscle tissue, which govern meat quality, and the variations thereof under the influence of the same factors are also addressed.

MICOL D., ROBELIN J., GEAY Y. 1993. Composition corporelle et caractéristiques biologiques des muscles chez les bovins en croissance et à l'engrais. INRA Prod. Anim., $6(1), 61$ - 69 . 